Portland State University

PDXScholar

Reduced Form for the General-state Multicenter Integral from an Integro-differential Transform

Jack C. Straton

Portland State University, straton@pdx.edu

Follow this and additional works at: https://pdxscholar.library.pdx.edu/phy_fac

Part of the Atomic, Molecular and Optical Physics Commons

Let us know how access to this document benefits you.

Citation Details

Straton, Jack C. "Reduced form for the general-state multicenter integral from an integro-differential transform." Physical Review A 41 (1990): 71-77.

This Article is brought to you for free and open access. It has been accepted for inclusion in Physics Faculty Publications and Presentations by an authorized administrator of PDXScholar. Please contact us if we can make this document more accessible: pdxscholar@pdx.edu. 


\title{
Reduced form for the general-state multicenter integral from an integro-differential transform
}

\author{
Jack C. Straton \\ Department of Physics, Cardwell Hall, Kansas State University, Manhattan, Kansas 66506
}

(Received 7 August 1989)

\begin{abstract}
In a previous paper Gaussian transforms were utilized to obtain the analytically reduced form for the class of multicenter integrals containing a product of hydrogenic orbitals for $s$-states, Yukawa or Coulomb potentials, and plane waves. In the present paper a related transformation is developed for nonspherical functions, leading to the reduced form for multicenter integrals that include hydrogenic orbitals representing states of arbitrary angular momentum.
\end{abstract}

\section{INTRODUCTION}

A central impediment to the evaluation of the merits of theories in atomic and molecular physics is the task of reducing the dimensionality of the multicenter integrals of a particular theory to a numerically tractable number. Direct spatial integration is not possible in general because the angular dependence in the coordinate-space functions appears sequestered in various square roots of quadratic forms. Researchers have commonly circumvented this problem by following the same general path.

(a) Introduce Fourier (three-dimensional integral) transforms ${ }^{1}$ for each of the terms in the product of orbitals and potentials. After evaluating the original (coordinate) integrals, and using the resulting $\delta$ functions to evaluate one or more of the (introduced) momentum integrals, all of the angular dependence resides in simple quadratic forms in exponentials and in denominators.

(b) Feynman (one-dimensional integral) transforms ${ }^{2}$ are then introduced for each term in the product of denominators to produce a quadratic form in a single denominator.

(c) One then completes the square in the denominator for each of the momentum integrals in turn, adjusting the exponentials accordingly, allowing that integral to be evaluated.

The author has attempted to make this generality of path explicit by carrying out these steps for a general multicenter integral. The Fourier transform has been found ${ }^{3}$ for a single-center product of hydrogenic orbitals representing arbitrary states and for a multicenter product of $1 s$ orbitals and Coulomb or Yukama potentials. ${ }^{4}$ For the latter, the iterative step c, was replaced by two steps.

$\left(c^{\prime}\right)$ An additional one-dimensional integral transform was introduced to move the Feynman denominator into an exponential so that all momentum dependence was contained within a single quadratic form.

(d) Finally, a unitary operator, ${ }^{5}$ that does not need to be explicitly found, was invoked to simultaneously diagonalize all momentum integrals so that they could be evaluated.

This approach was then used to find the reduced form for a multicenter integral (in any number of coordinates) of a product of $1 s$ hydrogenic orbitals, Coulomb or Yukawa potentials, and plane waves. ${ }^{6}$

But a fundamental limitation of the Fourier transform prevents the development of a "final reduced form," one free of derivatives to be evaluated, for multicenter integrals involving excited states, even those with $\ell=0$. Consider the Fourier integral representation ${ }^{7}$ of the $2 s$ hydrogenic orbital,

$$
u_{2 s}^{\lambda}(\mathbf{R})=\left(\frac{\lambda}{\pi}\right)^{5 / 2} \frac{1}{2^{3 / 2}} \int \frac{d^{3} k e^{i \mathbf{k} \cdot \mathbf{R}}\left(k^{2}-\lambda^{2} / 4\right)}{\left(\frac{\lambda^{2}}{4}+k^{2}\right)^{3}},
$$

where

$$
\lambda=\frac{Z}{a_{0}} .
$$

The polynomial in $k^{2}$ that appears in the numerator, which is typical of excited states, prevents one from using the above methods unless (1) is rewritten using a polynomial of derivatives. Setting $\gamma=\lambda / 2$,

$$
u_{2 s}^{\lambda}(\mathbf{R})=\frac{\lambda^{3 / 2}}{2^{3 / 2} \sqrt{\pi}}\left(1+\gamma \frac{\partial}{\partial \gamma}\right) e^{-\gamma R}
$$

which leads to

$$
u_{2 s}^{\lambda}(\mathbf{R})=\left(\frac{\lambda}{\pi}\right)^{5 / 2} \frac{1}{2^{3 / 2}}\left(1+\gamma \frac{\partial}{\partial \gamma}\right) \int d^{3} k \frac{e^{i \mathbf{k} \cdot \mathbf{R}}}{\left(\gamma^{2}+k^{2}\right)^{2}}
$$

One may then apply the general techniques outlined above, but the resulting expression contains derivatives of complicated functions left for the user to evaluate for his or her particular multicenter integral. Such an expression 
cannot be considered to be in "final form" in the sense that a result free of derivatives to be evaluated is final.

Fortunately, in a previous paper ${ }^{8}$ it was shown that Gaussian transforms ${ }^{9-15}$ may be used, in place of steps (a), (b), and ( $c^{\prime}$ ) above, to produce a quadratic form for step (d) to diagonalize.

Because the Gaussian transform is a one-dimensional integral, whereas the Fourier-Feynman transform is fourdimensional, one needs only to diagonalize with respect to the spatial variables. This gives a much simpler final reduced form than does the Fourier-Feynman transform in which both the spatial and the (introduced) momentum quadratic forms must be diagonalized.

However, the difference between these two transforms that is of a higher logical type than simple algebraic convenience is that the Gaussian transforms of $n s$ orbitals do not have the "pathologies" of Fourier transforms of these orbitals. If one examines the Gaussian transform of the "seed function" from which excited states are built by derivatives,

$$
\frac{e^{-\gamma R}}{R}=\frac{1}{\sqrt{\pi}} \int_{0}^{\infty} d \rho \frac{e^{-R^{2} \rho} e^{-\gamma^{2} / 4 \rho}}{\rho^{1 / 2}},
$$

it is seen that the parameter $\gamma$ that is used for the derivatives in (3) is decoupled (in the sense of differentiation) from the quadratic form $\rho R^{2}$ that will enter into the diagonalization. Then in the equivalent of (4),

$$
\begin{aligned}
u_{2 s}^{\lambda}(\mathbf{R}) & =\frac{\lambda^{3 / 2}}{2^{3 / 2} \sqrt{\pi}}\left(R-\gamma R^{2}\right) \frac{e^{-\gamma R}}{R} \\
& =\frac{\lambda^{3 / 2}}{2^{3 / 2} \pi}\left(-\frac{\partial}{\partial \gamma}-\gamma \frac{\partial^{2}}{\partial \gamma^{2}}\right) \int_{0}^{\infty} d \rho \frac{e^{-\rho R^{2}} e^{-\gamma^{2} / 4 \rho}}{\rho^{1 / 2}},
\end{aligned}
$$

one may evaluate the derivatives before diagonalizing and integrating over $d^{3} R$. Thus,

$$
\begin{aligned}
u_{2 s}^{\lambda}(\mathbf{R})=\frac{\lambda^{5 / 2}}{2^{7 / 2} \pi} \int_{0}^{\infty} & d \rho e^{-\rho R^{2}-\lambda^{2} / 16 \rho} \\
& \times\left(\frac{2}{\rho^{3 / 2}}-\frac{\lambda^{2}}{8 \rho^{5 / 2}}\right) .
\end{aligned}
$$

There is no such decoupling of the parameter $\gamma$, used in the derivatives, from the quadratic form in $k^{2}$ in the Fourier case (4).

In fact, the Gaussian transform for a spherically symmetric function

$$
P(R)=\int_{0}^{\infty} d \rho e^{-R^{2} \rho} A(\rho)
$$

is related to the Laplace transform ${ }^{9,14,15}$

$$
P(R)=G[A(\rho), R]=L\left[A(\rho) ; R^{2}\right],
$$

so that one may find the shape function $\mathrm{A}(\rho)$ that gives the correct integral transform of the object function $\mathrm{P}(\mathrm{R})$ from tables of inverse Laplace transforms, ${ }^{16}$

$$
A(\rho)=G^{-1}[P(R) ; \rho]=L^{-1}\left[P\left(s^{1 / 2}\right) ; \rho\right] \text {. }
$$

In the previous paper, ${ }^{8}$ the shape function for products of $s$-state hydrogenic orbitals and Coulomb or Yukawa potentials was found, which gave the analytically reduced form for multicenter integrals of such products. In the present paper the properties of solid harmonics are folded into the Gaussian transform to create an integrodifferential transform that places all coordinate dependence of an object function that may not be spherically symmetric into a single quadratic form in an exponential. This leads to the reduced form for a general multicenter integral that includes products of hydrogenic orbitals representing arbitrary states, Coulomb or Yukawa potentials, and plane waves.

\section{THE EXTENDED TRANSFORM}

The problem to be solved is the analytical reduction of the general class of integrals

$$
\begin{aligned}
S_{I_{1} J_{1} \cdots I_{M} J_{M}}^{\eta_{1} j_{1} \cdots \eta_{M} j_{M}}\left(\mathbf{p}_{1}, \ldots,\right. & \left.\mathbf{p}_{m} ; \mathbf{y}_{1}, \ldots, \mathbf{y}_{M}\right) \\
= & \int d^{3} x_{1} \cdots d^{3} x_{m} e^{-i\left(\mathbf{p}_{1} \cdot \mathbf{x}_{1}+\cdots+\mathbf{p}_{m} \cdot \mathbf{x}_{m}\right)} \\
& \quad \times P_{I_{1} J_{1}}^{\eta_{1} j_{1}}\left(\mathbf{R}_{i}\right) \cdots P_{I_{M} J_{M}}^{\eta_{M} j_{M}}\left(\mathbf{R}_{M}\right),(11)
\end{aligned}
$$

in which the arguments $\mathbf{R}$ are linear functions of one or more of the variables of integration and coordinate vectors that are external to the integral,

$$
\mathbf{R}_{i}=\sum_{j=1}^{m} t_{i j} \mathbf{x}_{j}+\sum_{j=1}^{M} u_{i j} \mathbf{y}_{j}
$$

In the object functions

$$
P_{I J}^{\eta j}(\mathbf{R})=u_{I}(\mathbf{R}) \cdots u_{J}(\mathbf{R}) V^{\eta j}(\mathbf{R})
$$

the $u$ 's are hydrogenic orbitals,

$$
u_{n \ell m}^{\lambda}(\mathbf{R})=\lambda^{3 / 2} N_{n \ell} F_{n \ell}(2 \lambda R / n) Y_{\ell m}(\hat{\mathbf{R}})
$$

where

$$
N_{n \ell}=\frac{2}{n^{2}} \sqrt{(n-\ell-1) !(n+\ell) !},
$$

and

$$
F_{n \ell}(2 \lambda R / n)=\sum_{s=0}^{n-\ell-1} \frac{(-1)^{s}(2 \lambda / n)^{s+\ell} R^{s+\ell} e^{-\lambda R / n}}{(n-\ell-1-s) !(2 \ell+1+s) ! s !} .
$$

The function

$$
V^{\eta j}(\mathbf{R})=R^{j-1} e^{-\eta R}
$$

is either a Coulomb potential $(j=0, \eta=0)$, a Yukawa potential $(j=0, \eta \neq 0)$, or $\sqrt{\pi} / \eta^{3 / 2}$ times a $1 s$ orbital $(j=1, \eta \neq 0)$. The usefulness of this $j$ index will become apparent in Sec. IV. Note that integrals in which $j<0$ require an independent derivation, from the 
present development of the reduced form, because the inverse Laplace transform on which the derivation would be based ${ }^{17}$ cannot be obtained from the shape function in (5) by differentiation.

The central task is to find an integral transformation for object functions such as (13) that contain nonspherical functions. Any function $P(\mathbf{R})$ may be expanded in (the complete set of) spherical harmonics, so this task reduces to finding an integral transformation that includes these functions. Solid harmonics

$$
\mathcal{Y}_{L M}(\mathbf{R}) \equiv R^{L} Y_{L M}(\hat{\mathbf{R}})
$$

have the property that ${ }^{18}$

$$
\mathcal{Y}_{L M}(\mathbf{R}) e^{-i \mathbf{k} \cdot \mathbf{R}}=\mathcal{Y}_{L M}\left(i \nabla_{k}\right) e^{-i \mathbf{k} \cdot \mathbf{R}} .
$$

If

$$
P(\mathbf{R})=\sum_{L, M} P_{L M}(R) \mathcal{Y}_{L M}(\mathbf{R})
$$

and if one may find the Gaussian shape functions

$$
A_{L M}(\rho)=G^{-1}\left[P_{L M}(R) ; \rho\right]=L^{-1}\left[P_{L M}\left(s^{1 / 2}\right) ; \rho\right]
$$

then one may define the shape functional

$$
A\left[\rho, i \nabla_{k}\right]=\sum_{L, M} A_{L M}(\rho) \mathcal{Y}_{L M}\left(i \nabla_{k}\right)
$$

such that

$$
P(\mathbf{R})=\left.\int_{0}^{\infty} d \rho A\left[\rho, i \nabla_{k}\right] e^{-\left(\rho R^{2}+i \mathbf{k} \cdot \mathbf{R}\right)}\right|_{\mathbf{k}=0} .
$$

This integro-differential transform has the desired form: All of the $\mathbf{R}$ dependence lies within a quadratic form in an exponential so that products of $P$ 's and plane waves may be written as an exponential of a single quadratic form to be diagonalized. In the present case it is found that after the diagonalization and integrating over the $R$ 's, the derivatives in (23) may be evaluated, leaving a shape function $A(\rho, \mathbf{q})$.

\section{THE REDUCED FORM OF THE MULTICENTER INTEGRAL}

In the present case, (13) contains a product of spherical harmonics for which (19) will not be of help since the solid harmonic gradient operator is not a linear operator in general. The angular momentum coupling must first be carried out. Define the shorthand notation

$$
\begin{aligned}
& \sum_{I, J} \circ Y_{L_{J} M_{J}}(\hat{\mathbf{R}}) \equiv Y_{\ell_{I} m_{I}}(\hat{\mathbf{R}}) \cdots Y_{\ell_{J} m_{J}}(\hat{\mathbf{R}}) \\
& =\sum_{L_{1}=L_{1} \min }^{L_{1} \max }(-1)^{M_{I+1}}\left(\frac{\left(2 \ell_{I}+1\right)\left(2 \ell_{I+1}+1\right)\left(2 L_{1}+1\right)}{4 \pi}\right)^{1 / 2}\left(\begin{array}{ccc}
\ell_{I} & \ell_{I+1} & L_{1} \\
0 & 0 & 0
\end{array}\right) \\
& \times\left(\begin{array}{ccc}
\ell_{I} & \ell_{I+1} & L_{1} \\
m_{I} & m_{I+1} & -M_{I+1}
\end{array}\right) \\
& \times \sum_{L_{2}=L_{2} \min }^{L_{2} \max }(-1)^{M_{I+2}}\left(\frac{\left(2 L_{1}+1\right)\left(2 \ell_{I+2}+1\right)\left(2 L_{2}+1\right)}{4 \pi}\right)^{1 / 2} \\
& \times\left(\begin{array}{ccc}
L_{1} & \ell_{I+2} & L_{2} \\
0 & 0 & 0
\end{array}\right)\left(\begin{array}{ccc}
L_{1} & \ell_{I+2} & L_{2} \\
M_{I} & m_{I+2} & -M_{I+2}
\end{array}\right) \times \cdots \\
& \times \sum_{L_{J}=L_{J} \min }^{L_{J} \max }(-1)^{M_{J}}\left(\frac{\left(2 L_{J-1}+1\right)\left(2 \ell_{J}+1\right)\left(2 L_{J}+1\right)}{4 \pi}\right)^{1 / 2} \\
& \times\left(\begin{array}{ccc}
L_{J-1} & \ell_{J} & L_{J} \\
0 & 0 & 0
\end{array}\right)\left(\begin{array}{ccc}
L_{J-1} & \ell_{J} & L_{J} \\
M_{J-1} & m_{J}-M_{J}
\end{array}\right) Y_{L_{J} M_{J}}(\hat{\mathbf{R}}),
\end{aligned}
$$

where

$$
\begin{aligned}
& M_{j}=m_{I}+m_{I+1}+\cdots+m_{j}, \\
& L_{j \max }=L_{j-1}+\ell_{j}, \\
& \mu_{j}=\max \left(\left|L_{j-1}-\ell_{j}\right|,\left|M_{j-1}+m_{j}\right|\right),
\end{aligned}
$$

and

$$
L_{j \min }=\left\{\begin{array}{l}
\mu_{j} \text { if } L_{j \max }+\mu_{j} \text { is even } \\
\mu_{j}+1 \text { if } L_{j \max }+\mu_{j} \text { is odd }
\end{array}\right.
$$


and where the index "(2)" on the summation sign indicates that one is to sum in steps of two. ${ }^{19}$ Then the shape functional corresponding to (13) is

$$
\begin{aligned}
A_{I J}^{\eta j}\left[\rho, i \nabla_{k}\right]=\frac{e^{-\gamma^{2} / 4 \rho}}{2^{j} \sqrt{\pi}}\left(\sum_{s_{I}=0}^{n_{I}-\ell_{I}-1} \frac{(-1)^{s_{I}}\left(\lambda_{I} / n_{I}\right)^{s_{I}+\ell_{I}} \lambda_{I}^{3 / 2} N_{n_{I} \ell_{I}}}{\left(n_{I}-\ell_{I}-1-s_{I}\right) !\left(2 \ell_{I}+1+s_{I}\right) ! s_{I} !}\right. \\
\left.\times \cdots \times \sum_{s_{J}=0}^{n_{J}-\ell_{J}-1} \frac{(-1)^{s_{J}}\left(\lambda_{J} / n_{J}\right)^{s_{J}+\ell_{J}} \lambda_{J}^{3 / 2} N_{n_{J} \ell_{J}}}{\left(n_{J}-\ell_{J}-1-s_{J}\right) !\left(2 \ell_{J}+1+s_{J}\right) ! s_{J} !}\right) \\
\times \sum_{I J} \circ\left(\frac{2^{L_{J}} H_{s_{I}+\ell_{I}+\cdots+s_{J}+\ell_{J}-L_{J}+j}(\gamma / 2 \sqrt{\rho})}{\rho^{\left(s_{I}+\ell_{I}+\cdots+s_{J}+\ell_{J}-L_{J}+1+j\right) / 2}} \mathcal{Y}_{L_{J} M_{J}}(i \nabla k)\right),
\end{aligned}
$$

where

$$
\gamma=\lambda_{I} / n_{I}+\cdots+\lambda_{J} / n_{J}+\eta
$$

and $\mathrm{H}$ is a Hermite polynomial.

The reduced form of the multicenter integral, equation (67) of the previous paper, which will be referred to as (67-I), may be generalized to include the arbitrary hydrogenic states in (11),

$$
S_{I_{1} J_{1} \cdots I_{M} J_{M}}^{\eta_{1} j_{1} \cdots \eta_{M} j_{M}}\left(\mathbf{p}_{1}, \ldots, \mathbf{p}_{m} ; \mathbf{y}_{1}, \ldots, \mathbf{y}_{M}\right)=\left.\pi^{3 m / 2} \int_{0}^{\infty} d \rho_{1} \cdots d \rho_{m} A_{I_{1} J_{1}}^{\eta_{1} j_{1}}\left[\rho_{1}, i \nabla_{k_{1}}\right] \cdots A_{I_{M} J_{M}}^{\eta_{M} j_{M}}\left[\rho_{M}, i \nabla_{k_{M}}\right] \frac{e^{-\omega / \Lambda}}{\Lambda^{3 / 2}}\right|_{\mathbf{k}_{\mathbf{\imath}}=0} .
$$

The only change is in the matrix $\mathrm{W}$ of $(28-\mathrm{I})$ in which each $\mathbf{b}_{\boldsymbol{i}}$, is replaced by

$$
\mathbf{B}_{i^{\prime}}=\mathbf{b}_{i^{\prime}}+\frac{i}{2} \sum_{j=1}^{M} t_{j i^{\prime}} \mathbf{k}_{j}
$$

where $\mathbf{b}_{i}(30-\mathrm{I})$, is

$$
\mathbf{b}_{i^{\prime}}=\frac{i p_{i^{\prime}}}{2}+\sum_{k=1}^{M} \rho_{k} t_{k i^{\prime}} \sum_{j=1}^{M} u_{k j} \mathbf{y}_{j} .
$$

The other elements of $\mathrm{W}(28-\mathrm{I})$ remain the same, except that $N+M \rightarrow M$. Equation (29-I) is

$$
a_{i j}=\sum_{k=1}^{M} \rho_{k} t_{k i} t_{k j}
$$

and $(31-\mathrm{I})$ is

$$
C=\sum_{k=1}^{M} \sum_{j=1}^{M} \sum_{j^{\prime}=1}^{M} \rho_{k} u_{k j} u_{k j^{\prime}} \mathbf{y}_{j} \cdot \mathbf{y}_{j^{\prime}},
$$

and the determinant $(36-\mathrm{I})$ is

$$
\Lambda=\left|\begin{array}{cccc}
a_{11} & a_{12} & \cdots & a_{1 m} \\
a_{21} & a_{22} & & a_{2 m} \\
\vdots & & \ddots & \vdots \\
a_{m 1} & a_{m 2} & \cdots & a_{m m}
\end{array}\right|
$$

Then the determinant (36-I) of $\mathrm{W}$ is

$$
\omega=\Omega-i \sum_{j=1}^{M} \mathbf{k}_{j} \cdot \mathbf{Q}_{j},
$$

where $\Omega(40-\mathrm{I})$ is as before,

$$
\Omega=C \Lambda+\sum_{i=1}^{m} \sum_{j=1}^{m} \mathbf{b}_{i} \cdot \mathbf{b}_{j}(-1)^{i+j+1} \Lambda_{i j},
$$

$\Lambda_{i j}$ is $\Lambda$ with the $i$ th row and $j$ th column deleted, and

$$
\begin{aligned}
\mathbf{Q}_{j}=-\sum_{i^{\prime}=1}^{m} \sum_{j^{\prime}=1}^{m} & (-1)^{i^{\prime}+j^{\prime}+1} \Lambda_{i^{\prime} j^{\prime}} \\
& \times\left(t_{j i^{\prime}} \mathbf{b}_{i^{\prime}}+\frac{i}{4} \sum_{\ell=1}^{M} t_{j i^{\prime}} t_{\ell j^{\prime}} \mathbf{k}_{\ell}\right) .
\end{aligned}
$$

Noting that

$$
i \frac{\partial}{\partial k_{j}^{s}}\left(-\frac{\omega}{\Lambda}\right)=-\frac{1}{\Lambda}\left(Q_{j}^{s}+\sum_{i^{\prime}=1}^{M} k_{i^{\prime}}^{r} \frac{\partial}{\partial k_{j}^{s}} Q_{i^{\prime} r}\right)
$$

all of the derivatives in (31) may be evaluated and the $k$ 's may be set to zero to give the final reduced form of the general multicenter integral,

$$
\begin{aligned}
& S_{I_{1} J_{1} I_{M} J_{M}}^{n_{1} j_{1} \cdots n_{M} j_{M}}\left(\mathbf{p}_{1}, \ldots, \mathbf{p}_{m} ; \mathbf{y}_{1}, \ldots, \mathbf{y}_{M}\right) \\
&=\pi^{3 m / 2} \int_{0}^{\infty} d \rho_{1} \cdots d \rho_{M} \\
& \times A_{I_{1} J_{1}}^{n_{1} j_{1}}\left(\rho_{1}, \mathbf{q}_{1}\right) \cdots A_{I_{M} J_{M}}^{n_{M} j_{M}}\left(\rho_{M}, \mathbf{q}_{M}\right) \\
& \times \frac{e^{-\Omega / \Lambda}}{\Lambda^{3 / 2}}
\end{aligned}
$$

where 


$$
\mathbf{q}_{i}=\frac{1}{\Lambda} \sum_{i^{\prime}=1}^{m} \sum_{j^{\prime}=1}^{m}(-1)^{i^{\prime}+j^{\prime}+1} \Lambda_{i^{\prime} j^{\prime}} t_{i i^{\prime}} \mathbf{b}_{i^{\prime}}
$$

and the shape functions $A(\rho, \mathbf{q})$ are given by the shape functional $A\left[\rho, i \nabla_{k}\right](29)$ by replacing the operator $i \nabla_{k}$ by the (c-number valued) vector $\mathrm{q}_{k}$.

\section{EXAMPLES}

In some cases (generally those in which no more than one $\mathbf{y}$ or $\mathbf{p}$ appears) the integrals in the "final" reduced form may be evaluated. Consider the easiest nontrivial case,

$$
S_{21 m \lambda}^{n j}(\mathbf{p} ; 0)=\int d^{3} x e^{-i \mathbf{p} \cdot \mathbf{x}} u_{21 m}^{\lambda}(\mathbf{x}) x^{j-1} e^{-\eta x}
$$

In this case

$$
\begin{aligned}
A_{21 m \lambda}^{\eta j}(\rho, \mathbf{q})= & \frac{e^{-\gamma^{2} / 4 \rho}}{2^{j} \sqrt{\pi}} \frac{\lambda^{3 / 2} 2 \sqrt{3} !}{2^{2}} \frac{\lambda / 2}{3 !} \frac{2^{1} H_{1-1+j}(\gamma / 2 \sqrt{\rho})}{\rho^{(1-1+1+j) / 2}} \\
& \times \mathcal{Y}_{1 m}(\mathbf{q}),
\end{aligned}
$$

where

$$
\mathbf{q}=\frac{-i \mathbf{p}}{2 \Lambda}
$$

and

$$
\gamma=\lambda / 2+\eta
$$

The determinants are

$$
\Lambda=\rho, \Lambda_{11} \equiv 1,
$$

and

$$
\Omega=\frac{p^{2}}{4} .
$$

Then

$$
\begin{aligned}
S_{21 m \lambda}^{\eta j}(\mathbf{p} ; 0)= & \frac{(2 \pi)^{3 / 2} \lambda^{5 / 2}}{2^{3+j} \sqrt{3 \pi}}\left(\frac{-i p}{2}\right) Y_{1 m}(\hat{\mathbf{p}}) \\
& \times \int_{0}^{\infty} d p \frac{e^{-\left(\gamma^{2}+p^{2}\right) / 4 \rho}}{\rho^{(6+j) / 2}} H_{j}\left(\frac{\gamma}{2 \sqrt{\rho}}\right) .
\end{aligned}
$$

Setting $j=1$,

$$
S_{21 m \lambda}^{\eta 1}(\mathbf{p} ; 0)=-\frac{(2 \pi)^{3 / 2} i \lambda^{7 / 2} 4 \gamma p Y_{1 m}(\hat{\mathbf{p}})}{\sqrt{3 \pi}\left(\gamma^{2}+p^{2}\right)^{3}}
$$

If $\eta=0,(50)$ is the nonsymmetrically normalized Fourier transform of a $2 p$ hydrogenic orbital, ${ }^{7}$

$$
\begin{aligned}
S_{21 m \lambda}^{01}(\mathbf{p} ; 0) & =(2 \pi)^{3 / 2} u_{21 m}^{\lambda}(\mathbf{p}) \\
& =(2 \pi)^{3 / 2}\left(\frac{-i 2 \lambda^{7 / 2} p Y_{1 m}(\hat{\mathbf{p}})}{\sqrt{3 \pi}\left(\frac{\lambda^{2}}{4}+p^{2}\right)^{3}}\right) .
\end{aligned}
$$

Setting $\eta=\lambda$ yields $\sqrt{\pi} / \lambda^{3 / 2}$ times the nonsymmetrically normalized Fourier transform of the single center product of $1 s$ and $2 p$ orbitals ${ }^{3}$

$$
\begin{aligned}
S_{21 m \lambda}^{\lambda 1}(\mathbf{p} ; 0) & =\frac{\sqrt{\pi}}{\lambda^{3 / 2}} I_{1 s, 2 p}(\mathbf{p}) \\
& =\frac{\sqrt{\pi}}{\lambda^{3 / 2}}\left(\frac{-i 4 \sqrt{6 \pi} \lambda^{5} p Y_{1 m}(\hat{\mathbf{p}})}{\left(9 \lambda^{2} / 4+p^{2}\right)^{3}}\right) .
\end{aligned}
$$

Finally, if $j=0,(49)$ gives

$$
S_{21 m \lambda}^{\eta 0}(\mathbf{p} ; 0)=\frac{-(2 \pi)^{3 / 2} i \lambda^{5 / 2} p Y_{1 m}(\hat{\mathbf{p}})}{\sqrt{3 \pi}\left(\gamma^{2}+p^{2}\right)^{2}}
$$

Now consider the more complicated integral

$$
\begin{aligned}
& S_{21-m \lambda^{\prime}, 21 m \lambda ; \mathbb{1}}^{\eta_{1} j_{1}, \eta_{2} j_{2}}(0 ; 0, \mathbf{y}) \\
& =\int d^{3} x\left[u_{21-m}^{\lambda^{\prime}}(\mathbf{x}) u_{21 m}^{\lambda}(\mathbf{x}) x^{j_{1}-1} e^{-\eta_{1} x}\right] \\
& \quad \times\left(|\mathbf{x}-\mathbf{y}|^{j_{2}-1} e^{-\eta_{2}|\mathbf{x}-\mathbf{y}|}\right)
\end{aligned}
$$

The first shape function is

$$
\begin{aligned}
& A_{21-m \lambda^{\prime}, 21 m \lambda}^{\eta_{1} j_{1}}\left(\rho_{1}, \mathbf{q}_{1}\right) \\
& \begin{array}{c}
=\frac{e^{-\gamma_{1}^{2} / 4 \rho_{1}}}{2^{j_{1}} \sqrt{\pi}} \frac{\left(\lambda^{\prime} \lambda\right)^{5 / 2}}{4}\left(\frac{2 \sqrt{3} !}{2^{2}}\right)^{2} \frac{1}{3 !^{2}} \sum_{L=0}^{2}{ }^{(2)} 3\left(\frac{2 L+1}{4 \pi}\right)^{1 / 2}\left(\begin{array}{ccc}
1 & 1 & L \\
0 & 0 & 0
\end{array}\right)\left(\begin{array}{ccc}
1 & 1 & L \\
-m & m & 0
\end{array}\right) \\
\times \frac{2^{L} H_{2-L+j_{1}}\left(\gamma_{1} / 2 \sqrt{\rho_{1}}\right)}{\rho_{1}^{\left(2-L+1+j_{1}\right) / 2}} \mathcal{Y}_{L 0}\left(\mathbf{q}_{1}\right) \\
=\frac{\left(\lambda^{\prime} \lambda\right)^{5 / 2}}{3 \times 2^{6+j_{1}} \pi} e^{-\gamma_{1}^{2} / 4 \rho_{1}}\left((-1)^{m} \mathcal{Y}_{00} \frac{H_{2+j_{1}}\left(\gamma_{1} / 2 \sqrt{\rho_{1}}\right)}{\rho_{1}^{\left(3+j_{1}\right) / 2}}+\frac{2^{2-|m|}}{\sqrt{5}} \mathcal{Y}_{20}\left(\mathbf{q}_{1}\right) \frac{H_{j_{1}}\left(\gamma_{1} / 2 \sqrt{p_{1}}\right)}{\rho_{1}^{\left(1+j_{1}\right) / 2}}\right)
\end{array}
\end{aligned}
$$


and the second is

$$
A_{\mathbb{1}}^{\eta_{1} j_{2}}\left(\rho_{2}, \mathbf{q}_{2}\right)=\frac{e^{-\eta_{2}^{2} / 4 \rho_{2}}}{2^{j_{2}} \sqrt{\pi}} \frac{H_{j_{2}}\left(\eta_{2} / 2 \sqrt{\rho_{2}}\right)}{\rho_{2}^{\left(1+j_{2}\right) / 2}} .
$$

Also

$$
\begin{aligned}
& a_{11}=\rho_{1}+\rho_{2} \equiv \Lambda, \\
& \mathbf{b}_{1}=-\rho_{2} \mathbf{y},
\end{aligned}
$$

$$
\begin{aligned}
& c=\rho_{2} y^{2}, \\
& \Omega=\rho_{1} \rho_{2} y^{2},
\end{aligned}
$$

and

$$
\mathbf{q}_{1}=\mathbf{q}_{2}=-\frac{\mathbf{b}_{1}}{\Lambda}=\frac{\rho_{2} \mathbf{y}}{\Lambda} .
$$

Consider the case $j_{1}=1, j_{2}=0$, then

$$
\begin{array}{r}
S_{21-m \lambda^{\prime}, 21 m \lambda ; \mathbb{1}}^{\eta_{1} 1 ; \eta_{2} 0}(0 ; 0, \mathbf{y})=\frac{\left(\lambda^{\prime} \lambda\right)^{5 / 2}}{3 \times 2^{7}} \int_{0}^{\infty} d \rho_{1} \int_{0}^{\infty} d \rho_{2}\left[\frac{(-1)^{m} \mathcal{Y}_{00} \gamma}{\rho_{1}^{2}}\left(\frac{\gamma^{3}}{\rho_{1}^{3 / 2}}-\frac{6 \gamma}{\rho_{1}^{1 / 2}}\right)+\frac{2^{2-|m|}}{\sqrt{5}}\left(\frac{\rho_{2}}{\Lambda}\right)^{2} \mathcal{Y}_{20}(\mathbf{y}) \frac{\gamma}{\rho_{1}^{3 / 2}}\right] \\
\\
\times e^{-\gamma^{2} / 4 \rho_{1}} e^{-\eta_{2}^{2} / 4 \rho_{2}} \frac{e^{-\rho_{1} \rho_{2} y^{2} / \Lambda}}{\Lambda^{3 / 2}}
\end{array}
$$

Changing variables from $\rho_{2}$ to

$$
\sigma=\left(\frac{\rho_{2}}{\Lambda}\right)^{1 / 2}
$$

and defining

$$
\mu^{2}=\gamma^{2}+\frac{\eta_{2}^{2}\left(1-\sigma^{2}\right)}{\sigma^{2}}
$$

one may reduce this to a one-dimensional integral ${ }^{20}$

$$
\begin{aligned}
S_{21-m \lambda^{\prime} 21 m \lambda ; \mathbb{1}}^{\eta_{1} 1 ; \eta_{2} 0}(0 ; 0, \mathbf{y})=\frac{\left(\lambda^{\prime} \lambda\right)^{5 / 2} \sqrt{\pi}}{3 \times 2^{7}} \int_{0}^{1} d \sigma\left\{(-1)^{m} \gamma \mathcal{Y}_{00}\right. & {\left[\frac{2^{7} \gamma^{3}}{\mu^{7 / 2}}\left(\frac{15}{4}+\frac{15}{4} \sigma y \mu+\frac{3}{2} \sigma^{2} y^{2} \mu^{2}+\frac{\sigma^{3} y^{3} \mu^{3}}{4}\right)\right.} \\
& \left.-\frac{3 \times 2^{6} \gamma}{\mu^{5 / 2}}\left(\frac{3}{2}+\frac{3 \sigma y \mu}{2}+\frac{\sigma^{2} y^{2} \mu^{2}}{2}\right)\right] \\
& \left.+\frac{2^{2-|m|}}{\sqrt{5}} \gamma \mathcal{Y}_{20}(\mathbf{y}) \frac{2^{3}}{\mu^{3 / 2}}\left(\sigma^{4}+y \sigma^{5} \mu\right)\right\} e^{-\sigma y \mu}
\end{aligned}
$$

Finally, for the special case $\eta_{2}=0$ (the Coulomb integral) $\mu \equiv \gamma$ and the analytic result is

$$
\begin{aligned}
& S_{21-m \lambda ; 21 m \lambda ; \mathbb{1}}^{\eta_{1} 1 ; 00}(0 ; 0, \mathbf{y}) \\
& =\frac{\left(\lambda^{\prime} \lambda\right)^{5 / 2}}{3 \times 2^{3} \gamma^{4}} \sqrt{\pi}\left\{\begin{array}{l}
(-1)^{m} Y_{00}\left[\frac{3 \times 2^{4}}{\gamma y}-\left(\frac{3 \times 2^{4}}{\gamma y}+3^{2} \times 2^{2}+3 \times 2^{2} \gamma y+2 \gamma^{2} y^{2}\right) e^{-\gamma y}\right] \\
\left.\quad+\frac{2^{2-|m|}}{\sqrt{5}} Y_{20}(\hat{\mathbf{R}})\left[\frac{3^{2} \times 2^{4}}{\gamma^{3} y^{3}}-\left(\frac{3^{2} \times 2^{4}}{\gamma^{3} y^{3}}+\frac{3^{2} 2^{4}}{\gamma^{2} y^{2}}+\frac{3^{2} \times 2^{3}}{\gamma y}+3 \times 2^{3}+3 \times 2 \gamma y+\gamma^{2} y^{2}\right) e^{-\gamma y}\right]\right\} .
\end{array}\right.
\end{aligned}
$$

This is the same result obtained ${ }^{21}$ using Fourier techniques. ${ }^{3}$

\section{CONCLUSION}

An integro-differential transform has been developed for functions $P(\mathbf{R})$ that may not be spherically symmetrical, in which all $\mathbf{R}$ dependence appears in a quadratic form in an exponential. This allows one to diagonalize the coordinate dependence of products of such functions and plane waves so that the $\mathrm{R}$ integrals may be evaluated.

The angular momentum coupling has been explicitly given for the case in which the $P$ 's are products of hydrogenic orbitals representing arbitrary states and Coulomb or Yukawa potentials. This integro-differential transform is then used to find the analytically reduced form for the general class of multicenter integrals of products of such $P$ 's with plane waves.

\section{ACKNOWLEDGMENTS}

This work was supported by the Division of Chemical Sciences, Office of Energy Research, U.S. Department of Energy. 
${ }^{1}$ The number of references using this technique is too large to list here. Examples of papers in which Fourier (momentum) techniques are prominently mentioned are H.P. Trivedi and E.O. Steinborn, Phys. Rev. A 27, 670 (1983); B.R. Junker, J. Phys. B 13, 1049 (1980); C. Guidotti, G.P. Arrighini, and F. Marinelli, Theor. Chim. Acta 53, 165 (1979); J. Avery and P. Ormen, Int. J. Quantum Chem. 18, 953 (1980); J. Grotendorst and E.O. Steinborn, J. Comput. Phys. 61, 195 (1985); A.W. Niukkanen, J. Math. Phys. 25, 698 (1984); E.J. Weniger, ibid. 26, 276 (1985); A.K. Bhattacharya and S.C. Dhabal, J. Chem. Phys. 84, 1598 (1986); E.J. Weniger and E.O. Steinborn, ibid. 87, 3709 (1987); J.S. Alper, ibid. 55, 3780 (1971), Eq. (18) with $R_{2}=0$ as corrected by K.G. Kay and J.S. Alper, ibid. 56, 4243 (1972); E.J. Weniger and E.O. Steinborn, J. Chem. Phys. 786121 (1983); E. Filter and E.O. Steinborn, Phys. Rev. A 18, 1 (1978), Eq. (2.14); A.W. Niukkanen, Int. J. Quantum. Chem. 25, 941 (1984); 25, 957 (1984); Y.B. Band, Phys. Rev. A 8, 243 (1973); B. Talukdar, J. Dutta, and H.P. Chattopadhay, J. Phys. B 17, 3211 (1984).

${ }^{2}$ R.P. Feynman, Phys. Rev. 76, 769 (1949).

${ }^{3}$ J.C. Straton, Phys. Rev. A 35, 2729 (1987); 37, 4531 (1988).

${ }^{4}$ J.C. Straton, Phys. Rev. A 39, 5062 (1989).

${ }^{5}$ J.S.R. Chisholm, Proc. Cambridge Philos. Soc. 48, 300 (1952).

${ }^{6}$ J.C. Straton, Phys. Rev. A 39, 5071 (1989).

${ }^{7}$ B. Podolsky and L. Pauling, Phys. Rev. 34, 109 (1929). This derivation is missing a $(-i)^{\ell}$ phase factor that is given correctly in E.J. Weniger, J. Math. Phys. 26, 276 (1985),
Eq. (4.30).

${ }^{8}$ J.C. Straton, Phys. Rev. A 39, 1676 (1989); 402819 (1989).

${ }^{9}$ R. Kikuchi, J. Chem. Phys. 22, 148 (1954), with $R=1$ and $\lambda \rightarrow R$.

${ }^{10}$ I. Shavitt and M. Karplus, J. Chem. Phys. 36, 550 (1962).

${ }^{11}$ J.P. Wright, Massachusetts Institute of Technology Solid State and Molecular Theory Group, Quarterly Progress Report No. 50, 1963 (unpublished).

${ }^{12}$ I. Shavitt and M. Karplus, J. Chem. Phys. 43, 398 (1965), Eq. (66).

${ }^{13}$ F.R. Burden and R.M. Wilson, Adv. Phys. 21, 825 (1972), p. 841.

${ }^{14}$ W.J. Taylor, J. Math. Phys. 19, 52 (1978).

${ }^{15}$ D.M. Bishop and R.L. Somorjai, J. Math. Phys. 11, 1150 (1970).

${ }^{16}$ A. Erdelyi, W. Magnus, F. Oberhettinger, and F.G. Tricomi, Tables of Integral Transforms (McGraw-Hill, New York, 1954), Vol. 1.

${ }^{17}$ A. Erdelyi, W. Magnus, F. Oberhettinger, and F. G. Tricomi, Ref. 16 , p. 246 , No. 9.

${ }^{18}$ B.F. Bayman, J. Math. Phys. 19, 2558 (1978).

${ }^{19}$ E.J. Weniger and E.O. Steinborn, J. Math. Phys. 26, 664 (1985).

${ }^{20}$ I.S. Gradshteyn and I.M. Ryzhik, Table of Integrals, Series, and Products (Academic, New York, 1980), p. 340, No. 3, 471(9); or derivatives with respect to $b$ of W. Grobner and N. Hofreiter, Integraltafel Zweiter Teil Bestimmte Interale (Springer-Verlag, Berlin, 1961) p. 66, No. 9c.

21 J.C. Straton and J.H. McGuire (unpublished). 